\title{
New Kinetic Theory and New Equations for Lattice and Electronic Heat Capacities, Enthalpies and Entropies of Solids: Application to Tungsten
}

\begin{abstract}
A.E. BOZDOĞAN*
Yıldız Technical University, Department of Chemistry, Davutpaşa, 34220 Esenler, Istanbul, Turkey

New equations for heat capacities, entropies and enthalpies were applied to the experimental constant volume and constant pressure heat capacity data of tungsten. The heat capacity $C_{P}$ max was predicted to be $24.96 \mathrm{~J} / \mathrm{molK}$ at $400 \mathrm{~K}$. The temperatures $\Theta_{V}$ and $\Theta_{P}$ were found to be $78.5 \mathrm{~K}$. The relationships between dimension and $\Theta_{V}$ and Debye temperature were given. Tungsten shows the dimensionality crossover from 3 to 2 at about $80 \mathrm{~K}$. Temperature dependences of Debye temperature, $\Theta_{V}$ and $\Theta_{P}$ and non-monotonic behavior were discussed. The heat capacity and entropy values predicted by the proposed models were compared with the values predicted by the Debye models. The results have shown that the proposed models fit the data better than the Debye models. The enthalpy values predicted by the proposed models were compared with the values predicted by the polynomial model and good fitting was obtained. The proposed models have shown that the constant pressure heat capacity data can be used to calculate enthalpies, entropies and heat capacities of tungsten.
\end{abstract}

DOI: 10.12693/APhysPolA.134.213

PACS/topics: heat capacity, entropy and enthalpy, dimension, tungsten

\section{Introduction}

Einstein introduced the first quantum statistical model with single oscillator for the temperature dependences of heat capacities of solids [1]. The values obtained from this model lie below the experimental heat capacities at low temperatures [2-4].

Nernst and Lindemann's two oscillator model has improved the fitting of heat capacity data in comparison with Einstein's model. However, this model could not well describe the heat capacities in the region $T<$ $100 \mathrm{~K}[5]$.

Einstein and Nernst-Lindemann's models have used the discrete oscillation frequencies, and the resulting theoretical curves obtained by these models show a plateau behavior in the $T \rightarrow 0 \mathrm{~K}$ limit. In the model given by Debye, atomic system is considered to be a three dimensional, elastic and isotropic continuum [6]. The heat capacity equation is given by $[2-4,6,7]$ :

$$
C_{V}=9 R\left(\frac{T}{\Theta_{\mathrm{D}}(T)}\right)^{3} \int_{0}^{x_{\mathrm{D}}} \frac{x^{4} \mathrm{e}^{x}}{\left(\mathrm{e}^{x}-1\right)^{2}} \mathrm{~d} x,
$$

where $\Theta_{\mathrm{D}}$ is Debye temperature, $x=\Theta_{\mathrm{D}}(T) / T$ and $R$ is gas constant.

The entropy equation in the Debye model is given by $[2,3,6]$ :

$$
S=3 R\left(\frac{4}{x_{\mathrm{D}}^{3}} \int_{0}^{x_{\mathrm{D}}} \frac{x^{3} \mathrm{~d} x}{\mathrm{e}^{x}-1}-\ln \left(1-\mathrm{e}^{-x_{\mathrm{D}}}\right)\right) .
$$

\footnotetext{
*e-mail: bozdogan@yildiz.edu.tr
}

The analytical solutions of integrals in Eq. (1) and Eq. (2) are not known. Therefore, at the intermediate temperatures, the values of heat capacities and entropies must be obtained by numerical integration.

At very low temperatures, where $T \ll \Theta_{\mathrm{D}}$, the following equation is obtained from Eq. (1):

$$
C_{V} \cong \frac{12 \pi^{4} R}{5}\left(\frac{T}{\Theta_{\mathrm{D}}(T)}\right)^{3} .
$$

Equation (3) is assumed to be valid from $0 \mathrm{~K}$ up to lattice temperatures of order $\theta_{\mathrm{D}}(0) / 50$, where $\Theta_{\mathrm{D}}(0)$ is the Debye temperature at $T \rightarrow 0 \mathrm{~K}$. $\Theta_{\mathrm{D}}$ depends on temperature. Therefore, it is often impossible to provide good fittings of Eq. (1) to the given heat capacity data sets with a single Debye temperature over the entire temperature range $[3,8]$. These non-Debye behaviors have been given in terms of $C_{V} / T^{3}$ functions [9-11]. These curves show a non-monotonic behavior in the low temperature region, which cannot be explained with the Debye's model.

The departure from Eq. (3) in the temperature interval $\theta_{\mathrm{D}}(0) / 50 \leq T \leq \theta_{\mathrm{D}}(0) / 10$ was assumed to be due to the deviation of frequency distribution. For this region, the equation based on Taylor series expansion has been proposed [3, 8, 12]. Different models based on Thirring and exponential series expansions have also been given for the intermediate to high temperature regions, respectively [5]. However, these models are more complex and seven or eight empirical parameters should be determined.

The heat capacity of a free electron gas in three dimensions for $T \ll T_{\mathrm{F}}$ has been given in [7]:

$$
C_{\mathrm{el}}=3 R \frac{\pi^{2}}{6} \frac{T}{T_{\mathrm{F}}}
$$

where $T_{\mathrm{F}}$ is the Fermi temperature. For high temperatures, the following equation has been given in [3]: 


$$
C_{\mathrm{el}}=\frac{3}{2} R z\left[1-\frac{1}{6(2 \pi)^{3 / 2}}\left(\frac{T_{\mathrm{F}}}{T}\right)^{3 / 2} \ldots\right],
$$

where $z$ is the number of free electrons per atom. For intermediate values of $\left(T / T_{\mathrm{F}}\right), C_{\mathrm{el}}$ must be computed numerically.

At low temperatures, the heat capacity of solids may be written as:

$$
C_{V}=\gamma T+A T^{3}
$$

where $\gamma$ and $A$ are characteristic constants of the solid [7]. A plot of $C_{V} / T$ versus $T^{2}$ should be a straight line with slope $A$ and intercept $\gamma$ and allows the determination of Debye temperature $\Theta_{D}(0)$ through $A=12 \pi^{4} R s / 5 \Theta_{\mathrm{D}}^{3}(0)$.

In this study, the heat capacity, enthalpy and entropy equations proposed in [13] will be applied to the constant volume and constant pressure heat capacity data of tungsten. The temperature and dimension dependence of Debye temperature and the deviation from monotonicity will be explained. The results obtained from the proposed Debye and polynomial equations will be compared.

\section{Heat capacity, entropy and enthalpy equations}

The heat capacity equation at constant volume,

$$
C_{V}=3 R \frac{T^{n}}{T^{n}+\Theta_{V}^{n}(T)},
$$

the heat capacity equation at constant pressure,

$$
C_{P}=C_{P \max } \frac{T^{n}}{T^{n}+\Theta_{p}^{n}(T)},
$$

and the electronic heat capacity equation

$$
C_{\mathrm{el}}=\frac{3}{2} R \frac{T^{n}}{T^{n}+T_{\mathrm{E}}^{n}(T)},
$$

were given in Ref. 13 .

If $n=3$, the following equation can be written at low temperature:

$$
C_{V}=3 R\left(\frac{T}{\Theta_{V}(T)}\right)^{3} .
$$

From Eq. (3) and Eq. (10), the following equation is obtained:

$$
\Theta_{V}(T)=\Theta_{\mathrm{D}}(T)\left(\frac{5}{4 \pi^{4}}\right)^{1 / 3} .
$$

The electronic molar entropy equation,

$$
S_{\mathrm{el}, n}=\frac{3}{2 n} R \ln \left(\left(\frac{T}{T_{\mathrm{E}}(T)}\right)^{n}+1\right),
$$

the lattice molar entropy equation at constant volume,

$$
S_{V, n}=\frac{3}{n} R \ln \left(\left(\frac{T}{\Theta_{V}(T)}\right)^{n}+1\right),
$$

and the lattice molar entropy equation at constant pressure

$$
S_{P, n}=\frac{C_{P \max }}{n} \ln \left(\left(\frac{T}{\Theta_{P}(T)}\right)^{n}+1\right),
$$

were given in Ref. 13 .
The lattice molar enthalpy equations at constant pressure for $n=1, n=2$ and $n=3$,

$$
\begin{gathered}
H_{p, n=1}=C_{P \max }\left(T+\Theta_{P} \ln \left(\frac{\Theta_{P}}{T+\Theta_{P}}\right)\right) \\
H_{p, n=2}=C_{P \max }\left(T-\Theta_{P} \cot \left(\frac{T}{\Theta_{P}}\right)\right) \\
H_{P, n=3}=C_{P \max }\left(-\frac{\pi}{6 \sqrt{3}} \Theta_{P}+T-\frac{\Theta_{P}}{3} \ln \left(\Theta_{P}+T\right)\right. \\
\left.+\frac{\Theta_{P}}{6} \ln \left(\Theta_{P}^{2}-\Theta_{P} T+T^{2}\right)-\frac{\Theta_{P}}{\sqrt{ } 3} \cot \left(\frac{2 T-\Theta_{P}}{\sqrt{ } 3 \Theta_{P}}\right)\right)
\end{gathered}
$$

the lattice molar enthalpy equations at constant volume for $n=1, n=2$ and $n=3$, and

$$
\begin{aligned}
& H_{V, n=1}=3 R\left(T+\Theta_{V} \ln \left(\frac{\Theta_{V}}{T+\Theta_{V}}\right)\right) \\
& H_{V, n=2}=3 R\left(T-\Theta_{V} \cot \left(\frac{T}{\Theta_{V}}\right)\right) \\
& H_{V, n=3}=3 R\left(-\frac{\pi}{6 \sqrt{3}} \Theta_{V}+T-\frac{\Theta_{V}}{3} \ln \left(\Theta_{V}+T\right)\right. \\
&\left.+\frac{\Theta_{V}}{6} \ln \left(\Theta_{V}^{2}-\Theta_{V} T+T^{2}\right)-\frac{\Theta_{V}}{\sqrt{ } 3} \cot \left(\frac{2 T-\Theta_{V}}{\sqrt{ } 3 \Theta_{V}}\right)\right),
\end{aligned}
$$

the electronic molar enthalpy equations for $n=1, n=2$ and $n=3$

$$
\begin{aligned}
& H_{\mathrm{el}, n=1}=\frac{3}{2} R\left(T+T_{\mathrm{E}} \ln \left(\frac{T_{\mathrm{E}}}{T+T_{\mathrm{E}}}\right)\right), \\
& H_{\mathrm{el}, n=2}=\frac{3}{2} R\left(T-T_{\mathrm{E}} \cot \left(\frac{T}{T_{\mathrm{E}}}\right)\right), \\
& H_{\mathrm{el}, n=3}=\frac{3}{2} R\left(-\frac{\pi}{6 \sqrt{3}} T_{\mathrm{E}}+T-\frac{T_{\mathrm{E}}}{3} \ln \left(T_{\mathrm{E}}+T\right)\right. \\
&\left.+\frac{T_{\mathrm{E}}}{6} \ln \left(T_{\mathrm{E}}^{2}-T_{\mathrm{E}} T+T^{2}\right)-\frac{T_{\mathrm{E}}}{\sqrt{ } 3} \cot \left(\frac{2 T-T_{\mathrm{E}}}{\sqrt{ } 3 T_{\mathrm{E}}}\right)\right),
\end{aligned}
$$

were given in Ref. 13.

\section{Results and discussion}

The heat capacity data of tungsten at constant volume and constant pressure for the temperature range from $1 \mathrm{~K}$ to $400 \mathrm{~K}$ were obtained from Ref. 14 and are shown in Fig. 1. The value of $C_{P \max }$ was predicted to be $24.96 \mathrm{~J} / \mathrm{molK}$ at $400 \mathrm{~K}$ from Eq. (8). The values of $\Theta_{V}$ and $\Theta_{P}$ were found to be $78.5 \mathrm{~K}$. The value of $\Theta_{\mathrm{D}}(0)$ was found to be $383 \mathrm{~K}$ from $C_{V}$ versus $T^{2}$ plot. The heat capacity values calculated using $\Theta_{\mathrm{D}}=383 \mathrm{~K}$ in Eq. (1) and the heat capacity values calculated by using $\Theta_{V}=78.5 \mathrm{~K}$ and $n=3$ in Eq. (7) are shown in Fig. 1.

The $\Theta_{\mathrm{D}}(T)$ values were calculated from the numerical solution of Eq. (1). Temperature dependence of $\Theta_{\mathrm{D}}(T)$ 


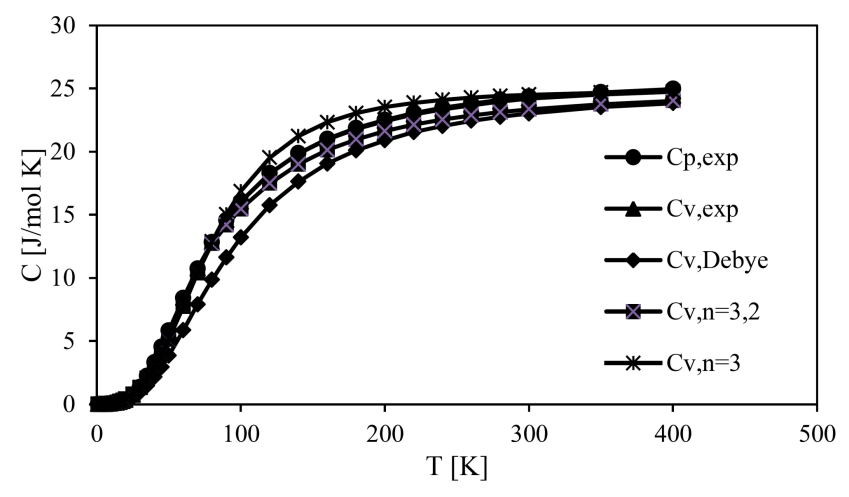

Fig. 1. Temperature dependence of heat capacity of tungsten.

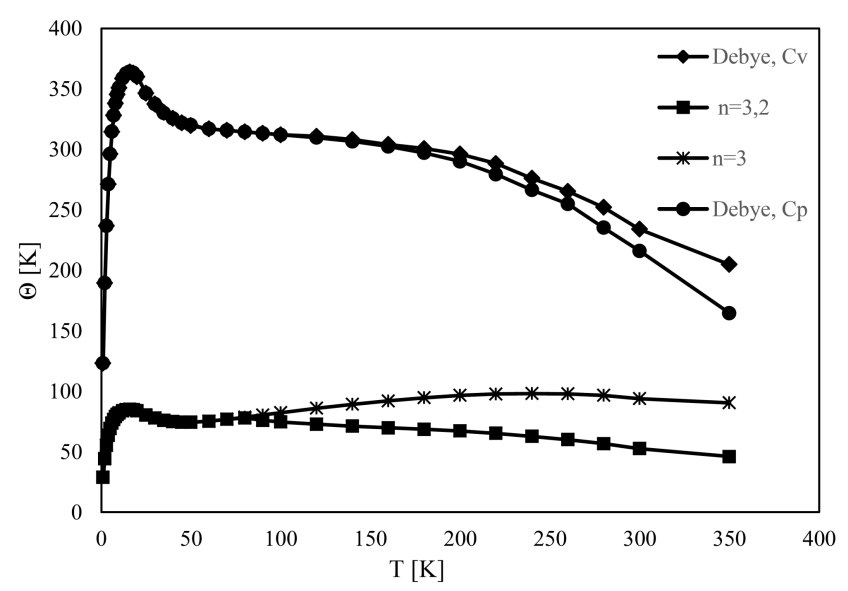

Fig. 2. Temperature dependence of $\Theta_{\mathrm{D}}(T)$ and $\Theta_{V}(T)$ of tungsten.

for tungsten is shown in Fig. 2. The value of $\Theta_{\mathrm{D}}(T)$ increases monotonously from $123 \mathrm{~K}$ to the maximum $364 \mathrm{~K}$ with increasing temperature from $1 \mathrm{~K}$ to $16 \mathrm{~K}$ and then decreases towards $165 \mathrm{~K}$ at about $350 \mathrm{~K}$.

The following equation is obtained from Eqs. (7) and (11):

$$
n=\frac{\log \left(\frac{3 R}{C_{V}}-1\right)}{\log \left(\Theta_{D(T)}\left(5 / 4 \pi^{4}\right)^{1 / 3} / T\right)} .
$$

The temperature and $\Theta_{\mathrm{D}}(T)$ dependence of $n$ for tungsten is shown in Fig. 3. The value of $n$ is about 3 from $1 \mathrm{~K}$ to $18 \mathrm{~K}$. It then decreases and passes through a minimum of 2.92 at about $40 \mathrm{~K}$ and then increases to 3 at $50 \mathrm{~K}$. $n$ exhibits a crossover from 3 to 2 at about $80 \mathrm{~K}$. After $80 \mathrm{~K}, n$ takes the value of about 2 . Figure 2 and Eq. (24) show that $\Theta_{\mathrm{D}}(T)$ depends on temperature and $n$.

The following equation is obtained from Eq. (7):

$$
\Theta_{V}(T)=T\left(\frac{3 R}{C_{V}}-1\right)^{1 / n}
$$

The $\Theta_{V}(T)$ values were calculated by using $n=3$ at all temperatures, and by using $n=3$ from $1 \mathrm{~K}$ to $80 \mathrm{~K}$ and

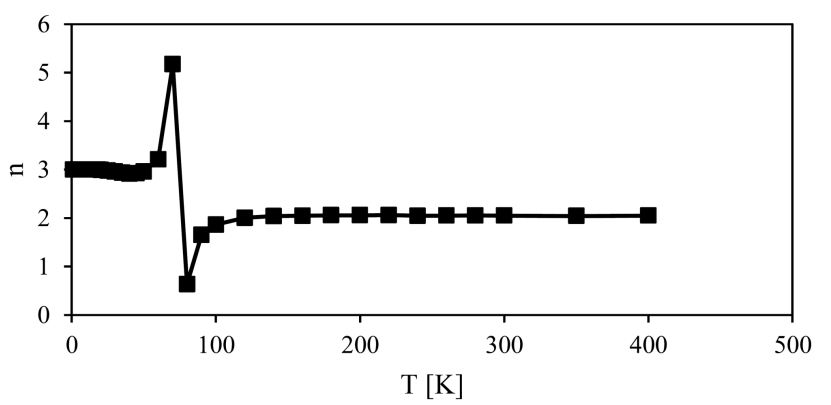

Fig. 3. Temperature dependence of $n$ of tungsten.

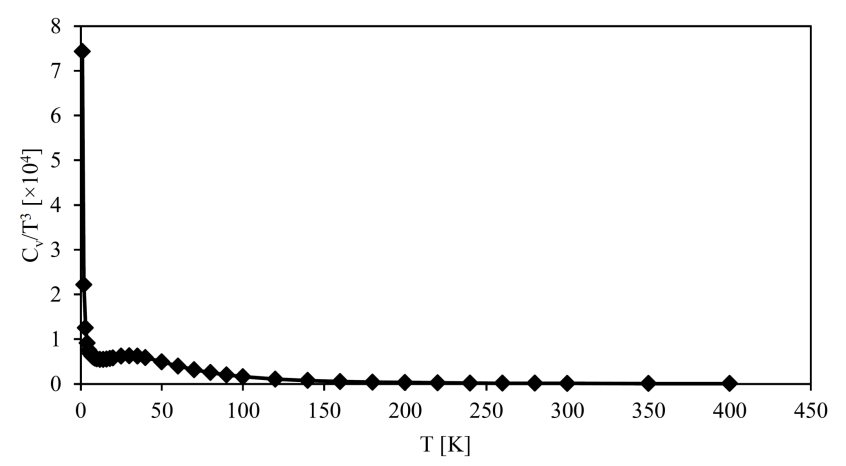

Fig. 4. $\quad C_{V} / T^{3}$ versus $T$ for tungsten.

by using $n=2$ from $80 \mathrm{~K}$ to $400 \mathrm{~K}$ in Eq. (25). Figure 2 shows that the temperature dependence of $\Theta_{V}(T)$, obtained by using $n=3$ from $1 \mathrm{~K}$ to $80 \mathrm{~K}$ and by using $n=2$ from $80 \mathrm{~K}$ to $400 \mathrm{~K}$, is similar to $\Theta_{\mathrm{D}}(T)$. However, the $\Theta_{V}(T)$ values obtained by using $n=3$ at all temperatures are different.

Figure 4 shows the non-monotonic behavior of the $C_{V} / T^{3}$ function at low temperatures. $n$ was taken to be 3 in this function. It is seen from Eqs. (3) and (10) that the $C_{V} / T^{3}$ is inversely proportional to $\Theta_{\mathrm{D}}^{3}(T)$ and $\Theta_{V}^{3}(T)$. Therefore, the $C_{V} / T^{3}$ function shows the inverse behavior to $\Theta_{\mathrm{D}}^{3}(T)$ and $\Theta_{V}^{3}(T)$.

The root mean square error of prediction (RMSEP) is obtained from the following equation:

$$
\operatorname{RMSEP}(C)=\sqrt{\frac{1}{m} \sum_{i=1}^{m}\left(C_{V \text { pred }}-C_{V \text { exp }}\right)^{2}},
$$

where $C_{\exp }$ is the experimental heat capacity at constant volume and constant pressure, $C_{\text {pred }}$ is the predicted heat capacity at constant volume and constant pressure and $m$ is the number of heat capacities. The values of RMSEP obtained for the proposed models by using $\Theta_{V}(T)=\Theta_{P}(T)=78.5 \mathrm{~K}$ and $n=3$ from $1 \mathrm{~K}$ to $80 \mathrm{~K}$ and $n=2$ from $80 \mathrm{~K}$ to $400 \mathrm{~K}$ were found to be 0.5063 and 0.5654 for constant volume and constant pressure heat capacities, respectively. The value of RMSEP obtained for the Debye model was found to be 1.437 . These results show that the proposed model obtained by using $n=3$ from $1 \mathrm{~K}$ to $80 \mathrm{~K}$ and $n=2$ from $80 \mathrm{~K}$ to $400 \mathrm{~K}$ fits the experimental data better than the other 
models and tungsten exhibits dimensionality crossover from 3 to 2 at about $80 \mathrm{~K}$.

The values of entropies of tungsten obtained from the Debye model, the proposed and the polynomial models are shown in Fig. 5 .

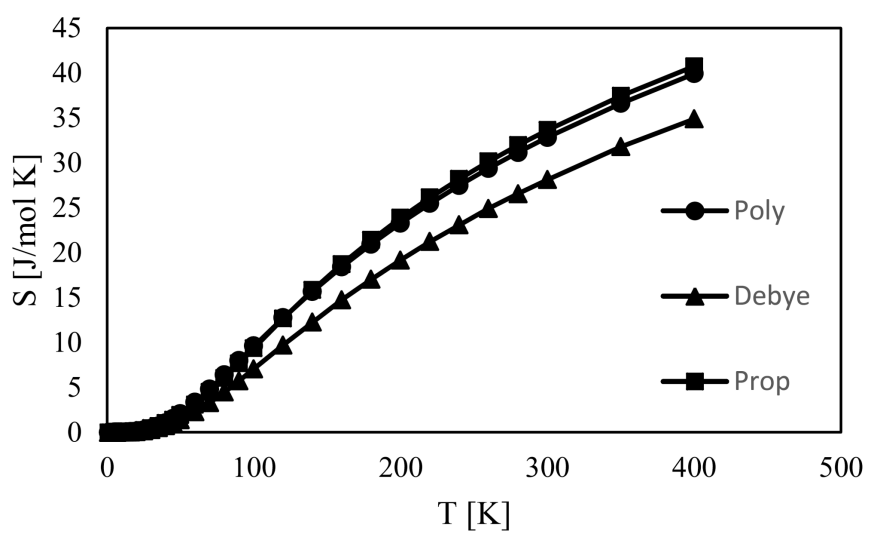

Fig. 5. Temperature dependence of entropy of tungsten.

The RMSEP values for entropy are obtained from the following equation:

$$
\operatorname{RMSEP}(S)=\sqrt{\frac{1}{m} \sum_{i=1}^{m}\left(S_{\text {pred }}-S_{\text {poly }}\right)^{2}},
$$

where $S_{\text {poly }}$ is the entropy obtained from the polynomial model and $S_{\text {pred }}$ is the entropy obtained from the proposed and the Debye models. The values of RMSEP obtained for the proposed and the Debye models were found to be 0.3795 and 2.4858 , respectively. These results and Fig. 5 show that the proposed model fits the data better than the Debye model.

Enthalpy equation cannot be obtained from the Debye model. The enthalpy values of tungsten obtained from the proposed and polynomial models are shown in Fig. 6 .

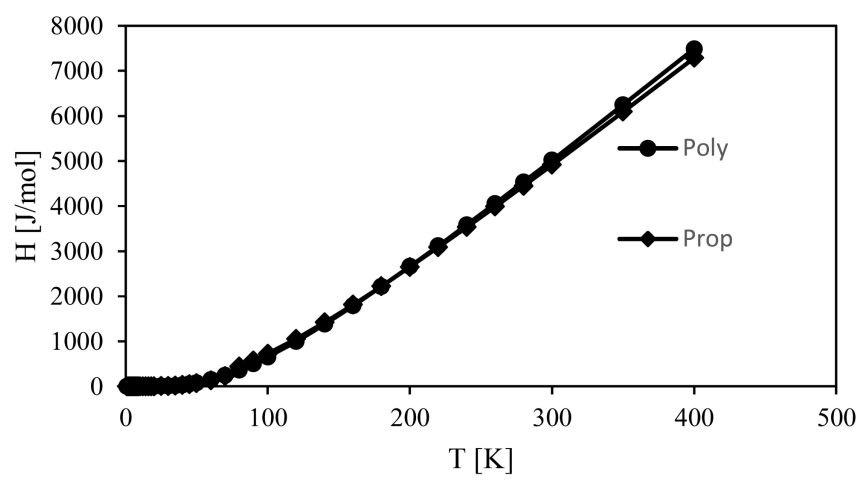

Fig. 6. Temperature dependence of enthalpy of tungsten.

The RMSEP value for enthalpy is obtained from the following equation:

$$
\operatorname{RMSEP}(H)=\sqrt{\frac{1}{m} \sum_{i=1}^{m}\left(H_{\text {pred }}-H_{\text {poly }}\right)^{2}},
$$

where $H_{\text {poly }}$ is the enthalpy obtained from polynomial model and $H_{\text {pred }}$ is the enthalpy obtained from the proposed model. The value of RMSEP was found to be 54.71. This result and Fig. 6 show that the proposed model fits the data very well. The value of $\Theta_{V}(T)$ is equal to the value of $\Theta_{P}(T)$ and $C_{P \max }$ is approximately equal to $3 R$. Therefore, the RMSEP values of entropy and enthalpy obtained for constant volume and constant pressure are approximately equal to each other.

\section{Conclusions}

The values of $\Theta_{V}$ and $\Theta_{P}$ were found to be $78.5 \mathrm{~K}$ for tungsten. Tungsten shows the dimensionality crossover from $n=3$ to $n=2$ at about $80 \mathrm{~K}$. The temperature and $n$ dependence of $\Theta_{\mathrm{D}}(T)$ and the non-monotonic behavior were discussed. The relationship between $n$ and $\Theta_{V}(T)$ was given. The heat capacity and entropy values predicted by the proposed models were compared with the values predicted by the Debye models. The results have shown that the proposed models fit the data better than the Debye models. The enthalpy values predicted by the proposed models were compared with the values predicted by the polynomial model.

\section{References}

[1] A. Einstein, Annals Phys. 22, 180 (1907).

[2] E.S.R. Gopal, Specific Heats at Low Temperatures, Plenum Press, New York 1966, p. 25.

[3] A. Tari, The Specific Heat of Matter at Low Temperatures, Imperial Collage Press, London 2003, p. 19.

[4] D.W. Rogers, Einstein's Other Theory. The PlanckBose Theory of Heat Capacity, Princeton University Press, 2005 p. 33.

[5] R. Passler, Phys. Status Solidi B 245, 1133 (2008).

[6] P. Debye, Annals Phys. 39, 789 (1912).

[7] C. Kittel, Introduction to Solid State Physics, Wiley, New York 2005, p. 115.

[8] R. Passler, Phys. Status Solidi B 247, 77 (2010).

[9] M. Sanati, S.K. Estreicher, M. Cardona, Solid State Commun. 131, 229 (2004).

[10] A. Gibin, G.G. Devyathykh, A.V. Gusev, R.K. Kramer, M. Cardona, H.-J. Pohl, Solid State Commun. 133, 569 (2005).

[11] M. Cardona, R.K. Kramer, M. Sanati, S.K. Estreicher, T.R. Antony, Solid State Commun.133, 465 (2005).

[12] P. Flubacher, A.J. Leadbetter, J.A. Morrison, Phil. Mag. 4, 273 (1959).

[13] A.E. Bozdoğan, Acta Physica Polonica A 131, 519 (2017).

[14] G.K. White, S.J. Collocott, J. Phys. Chem. Ref. Data 13, 1251 (1984). 